\title{
PLATELET-RICH FIBRIN SECRETOME INDUCES THREE DIMENSIONAL ANGIOGENIC ACTIVATION IN VITRO
}

\author{
C. Herrera-Vizcaíno ${ }^{1, \$}$, E. Dohle ${ }^{1, \$}$, S. Al-Maawi ${ }^{1}$, P. Booms ${ }^{1}$, R. Sader ${ }^{1}$, C.J. Kirkpatrick ${ }^{1}$, \\ J. Choukroun ${ }^{1,2}$ and S. Ghanaati ${ }^{1, *}$ \\ ${ }^{1}$ FORM, Frankfurt Oral Regenerative Medicine, Clinic for Maxillofacial and Plastic Surgery, Johann Wolfgang \\ Goethe University, Frankfurt am Main, Germany \\ ${ }^{2}$ Private practice, Pain Therapy Centre, Nice, France \\ $\S$ These authors contributed equally
}

\begin{abstract}
Different tissue engineering techniques are used to support rapid vascularisation. A novel technique is the use of platelet-rich fibrin (PRF), an autologous source of growth factors. This study was the first to investigate the influence of PRF matrices, isolated following different centrifugation protocols, on human dermal vascular endothelial cells (ECs) in mono-culture and co-culture with human primary fibroblasts (HFs) as an in vitro model for tissue regeneration. Focus was placed on vascular structure formation and growth factor release. HFs and ECs were cultivated with PRF prepared using a high $(710 \times g)$ or low $(44 \times g)$ relative centrifugation force (RCF) over $14 \mathrm{~d}$. Immunofluorescence staining and immunohistochemistry were used to evaluate the microvascular formation. Cell culture supernatants were collected for evaluation of growth factor release. The results showed a PRF-mediated effect on the induction of angiogenesis in ECs. Microvessel-like structure formation was promoted when ECs were combined with low-RCF PRF as compared to high-RCF PRF or control group. The percentage of vascular lumen area was significantly higher in low-RCF PRF, especially at day 7, which coincided with statistically significantly higher growth factor [vascular endothelial factor (VEGF), transforming growth factor $\beta 1$ (TGF- $\beta 1$ ) and platelet derived growth factor (PDGF)] concentration measured in low-RCF PRF as compared to high-RCF PRF or control group. In conclusion, reducing the RCF according to the low-speed centrifugation concept (LSCC) resulted in increased growth factor release and angiogenic structure formation with EC mono-culture, suggesting that PRF may be a highly beneficial therapeutic tool for tissue engineering applications.
\end{abstract}

Keywords: Platelet-rich fibrin, low speed centrifugation concept, angiogenesis, secretome.

*Address for correspondence: Shahram Ghanaati, FORM, Frankfurt Oral Regenerative Medicine, Clinic for Maxillofacial and Plastic Surgery, Johann Wolfgang Goethe University, Frankfurt am Main, Germany. Telephone number: +496963013744 Email: Shahram.Ghanaati@kgu.de

Copyright policy: This article is distributed in accordance with Creative Commons Attribution Licence (http://creativecommons.org/licenses/by-sa/4.0/).

\section{Introduction}

Rapid vascularisation of engineered tissues is necessary for regeneration of tissue defects, caused by trauma or tumour resection, since the vasculature supplies cells with nutrients and oxygen (Auger et al., 2013; Liu et al., 2017). For this reason, one of the main goals in the fields of tissue engineering and regenerative medicine is the generation of tissues that can be used in place of autologous tissues. For optimal integration, engineered tissues need to possess several features; the inability to achieve sufficient vascularisation is one of the key limitations hindering the translation of regenerative technologies from bench to bedside. Different tissue engineering approaches, such as the use of skin and bone substitute materials, have been developed to support bone and soft tissue regeneration. Therefore, various concepts have been implemented to obtain tissue-like constructs, i.e. pre-cultured biomaterials containing living cells to facilitate tissue vascularisation. In previous studies, "biologisation" of acellular biomaterials prior to their implantation in vivo has improved their integration in the application region (Al-Maawi et al., 2017; Barbeck et al., 2015). In this context, biomaterials that are precultured using human osteoblasts and endothelial cells undergo significantly higher vascularisation 
in vivo as compared to pure biomaterials (Fuchs et al., 2009; Ghanaati et al., 2011; Unger et al., 2010). Barbeck et al. (2016) demonstrated the positive effect of monocytes isolated from the peripheral blood on vascularisation. The isolated monocytes were cultured on a bone substitute material prior to its subcutaneous implantation in vivo. The addition of peripheral blood monocytes to the biomaterial results in a higher rate of vascularisation when compared to the untreated biomaterial. In addition, peripheral blood neutrophils have a significantly positive effect on angiogenesis and osteogenesis in vitro (Herath et al., 2018). Nevertheless, these strategies require cell isolation and, in some cases, cell growth and expansion for 3-4 weeks prior to biomaterial implantation, a protocol that currently hinders translation to the clinics.

In the context of a clinically applicable method, platelet-rich fibrin (PRF) has been introduced as a beneficial tool to enhance tissue regeneration (Choukroun et al., 2006a; Choukroun et al., 2006b; Choukroun, 2001; Dohan et al., 2006a; Dohan et al., 2006b; Dohan et al., 2006c; Ghanaati et al., 2014). This blood concentration-system involves centrifugation of the patient's own peripheral blood in a single step. According to the protocol implemented, it is possible to obtain solid as well as injectable liquid PRF matrices without the requirement of additives such as anticoagulants (Choukroun and Ghanaati, 2018). The resulting PRF contains fibrin, platelets, leukocytes and plasma proteins. Furthermore, Ghanaati et al. (2014) showed that it could function as a tailored drug delivery system. Based on the lowspeed centrifugation concept (LSCC), Choukroun and Ghanaati (2018) reported the influence of systematically altering the applied relative centrifugal force (RCF) on cell number and growth factor release within the injectable PRF. The number of leukocytes, neutrophils, monocytes, lymphocytes and platelets is significantly higher in PRF matrices prepared using low RCF as compared to those prepared using high RCF. Additionally, the total growth factor release of platelet-derived growth factor (PDGF)-BB, transforming growth factor $\beta 1$ (TGF- $\beta 1$ ), endothelial growth factor (EGF), vascular endothelial factor (VEGF) and matrix metallopeptidase 9 (MMP-9) is significantly higher in the low RCF group than that in the high RCF group (Wend et al., 2017).

Relevant in vitro models include the use of human primary fibroblasts (HFs) and endothelial cells (ECs) embedded in three-dimensional (3D) substrates (DeCicco-Skinner et al., 2014; Liu et al., 2017). A variety of endothelial culture media are reported (Price, 2017) to be enriched with varying concentrations of serum and synthetic growth factors, such as VEGF, fibroblast growth factor (FGF), insulin growth factor (IGF) and EGF. Although the methods mentioned previously provide conditions for the formation of vascular structures, the synthetic combination of growth factors (GFs) in a culture medium lacks the complexity and repertoire of proteins secreted naturally (secretome) into the extracellular space (Veronesi et al., 2017). The role of the secretome, i.e. all the factors released by the cells within the physiological environment, in tissue regeneration is gaining interest as the best method to recapitulate the broad beneficial effects of paracrine mechanisms. The secretome includes trophic factors (chemokines, cytokines, GFs, hormones and lipid mediators) with the capacity to affect receptor-mediated survival pathways, differentiation, pro- and anti-inflammatory cell behaviour and wound healing (Burdon et al., 2011; Mukherjee and Mani, 2013). Considering PRF as a natural source of growth factors, Dohle et al. (2017) demonstrated the effectiveness of injectable PRF for inducing vascular formation and osteogenic differentiation in vitro. In this context, co-cultures of outgrowth endothelial cells (OECs) and primary osteoblasts incubated in injectable PRF clots produce significantly more microvessel-like structures as compared with co-cultures on cell culture plastic. These results suggest a pro-angiogenic property of injectable PRF in the in vitro co-culture model.

The purpose of the present study was to investigate the influence of PRF-matrices prepared using high $(710 \times g)$ or low $(44 \times g)$ RCF on human dermal vascular endothelial cells (HDMECs) in monoculture and in co-culture with HFs. The final aim was to implement an in vitro model system for soft tissue regeneration in which vascular structure formation and growth factor secretion could be studied. Reducing the time of cell culturing to that required to induce the process of angiogenesis would facilitate the translation to the clinical environment of numerous cell-based tissue engineering techniques. Furthermore, this study may provide basic evidence that PRF could serve as "biologisation" tool of acellular biomaterials as a clinically applicable concept.

\section{Materials and Methods}

\section{Isolation and cultivation of primary cells}

HFs and HDMECs were isolated from excesstissue samples of patients undergoing cleft lip reconstruction, according to a standard protocol (Unger et al., 2002). The surgeries occurred in the plastic and maxillofacial surgery service of the University Hospital of Frankfurt, Germany. The acquisition and application of all cells that were used in the study were in accordance with the principle of informed consent and approved by the responsible Ethics Commission of the state of Hessen, Germany. ECs were cultivated at $37{ }^{\circ} \mathrm{C}$ and $5 \% \mathrm{CO}_{2}$ in $75 \mathrm{~cm}^{2}$ flasks with Endothelial Cell Growth Basal Medium-2 (EBM $^{\mathrm{TM}}-2$; Lonza), supplemented with EGM ${ }^{\mathrm{TM}}-2$ Endothelial Cell Growth Medium-2 BulletKit ${ }^{\mathrm{TM}}$ (Lonza), $1 \%$ penicillin/streptomycin (Sigma-Aldrich) and $4 \%$ foetal bovine serum (Biochrom). HFs were grown and cultivated in $75 \mathrm{~cm}^{2}$ flasks using Dulbecco's modified minimum essential medium (DMEM) medium (Biochrom), supplemented with 
Table 1. Different PRF protocols generated by different RCFs. Based on the LSCC, high $(710 \times g)$ and low-RCF $(44 \times g)$ centrifugation protocols were selected for comparison in the study.

\begin{tabular}{|c|c|c|c|c|c|c|}
\hline PRF protocol & $\begin{array}{c}\text { Rotation } \\
\text { (rpm) }\end{array}$ & Time (min) & $\begin{array}{c}\text { g-force } \\
\text { (RCF) }\end{array}$ & $\begin{array}{c}\text { Tube } \\
\text { material }\end{array}$ & Radius (mm) & Centrifuge \\
\hline PRF low & 600 & 8 & 44 & plastic & 110 & $\begin{array}{c}\text { Duo } \\
\text { centrifuge }\end{array}$ \\
\hline PRF high & 2,400 & 8 & 710 & plastic & 110 & $\begin{array}{c}\text { Duo } \\
\text { centrifuge }\end{array}$ \\
\hline
\end{tabular}

$1 \%$ penicillin/streptomycin (Sigma-Aldrich) and $4 \%$ foetal bovine serum (Biochrom). For the study, ECs were trypsinised and expanded until passage 4 and HFs until passage 7 .

\section{PRF-LSCC}

PRF was obtained from 3 healthy donors and each donor was used independently. Blood was collected from the antecubital vein using a vacuum bloodcollection butterfly. The blood was immediately transferred into $10 \mathrm{~mL} \mathrm{i-PRF}{ }^{\circledR}$ tubes (Process for PRF, Nice, France) and centrifuged in a Duo ${ }^{\circledR}$ centrifuge (Process for PRF). Based on the LSCC, a high $(710 \times g)$ and a low-RCF $(44 \times g)$ centrifugation protocol were selected for comparison (Table 1). After centrifugation, 2 layers could be observed: the red blood cells at the bottom and the injectable PRF at the top, containing fibrin, platelets, leukocytes and plasma proteins. Although the i-PRF ${ }^{\circledR}$ tubes do not contain anti-coagulants, PRF did not clot immediately, but after 10 to $15 \mathrm{~min}$ following centrifugation and depending on the donor. The layers of the generated injectable PRF which were still liquid after centrifugation were immediately aspirated from all tubes using a syringe. Low- and high-RCF PRF were homogenised separately for each donor in $15 \mathrm{~mL}$ tubes. The resulting homogenised PRF was used for cell culture experiments as described in the following section. As control group, $1 \mathrm{~mL}$ of high- and low-RCF PRF, respectively, was cultivated alone with $1 \mathrm{~mL}$ of $\mathrm{EBM}^{\mathrm{TM}}-2$.

\section{Cell culture experiments}

Primary cells were isolated from excess tissue of infant patients undergoing cleft lip reconstruction and PRF was generated from 3 additional human donors. The cell culture experiments were conducted in triplicate in 24-well plates. In the experimental groups, a total of $1 \mathrm{~mL}$ of high-RCF or low-RCF PRF was deposited in each well for 20 min until clotted; in the control group, $1 \mathrm{~mL}$ of collagen type I from rat tail (Sigma-Aldrich) was deposited in the wells. On top of the collagen and the clotted PRF, 20,000 cells per well (HFs or ECs) were seeded for mono-culture; for co-culture, 10,000 HFs and ECs, respectively, were seeded per well. Cells were resuspended in $1 \mathrm{~mL}$ of $\mathrm{EBM}^{\mathrm{TM}}-2$ enriched with $\mathrm{EGM}^{\mathrm{TM}}-2$ kit supplements. The individual experimental groups are indicated in Table 2. After 7 or $14 \mathrm{~d}$ of cultivation, cells were fixed in formaldehyde for immunofluorescence staining and immunohistochemistry. Cell culture supernatants were collected after 4, 7 and $14 \mathrm{~d}$ and replaced with fresh medium. The collected supernatants were assayed for growth factor concentration by enzymelinked immunosorbent assay (ELISA).

\section{Protein quantification by ELISA}

Quantification of growth factors within the cell culture supernatants and from PRF cultivated alone was performed using quantitative sandwich ELISA. Briefly, the release of TGF- $\beta 1$, VEGF and PDGF-BB was quantified using the DuoSet ELISA kit (R\&D Systems) following the manufacturer's instructions. The absorbance assay was conducted using a microplate reader (Infinite M200, Tecan, Grödig, Austria) set to a wavelength of $450 \mathrm{~nm}$ with a reference reading at $570 \mathrm{~nm}$. The output data were analysed using a 4-parameter logistic curve fit and presented graphically using Graph-Pad Prism version 6.0 (GraphPad Software).

\section{Immunofluorescence staining and immunohistochemistry}

For immunofluorescence staining, cell culture wells were fixed in formaldehyde $4 \%$ for $10 \mathrm{~min}$ and cells were washed three times with phosphate-buffered saline (PBS). Cells were permeabilised for $5 \mathrm{~min}$ using $0.1 \%$ Triton- $X$ in PBS, followed by incubation with mouse anti-human CD-31 antibody (1:40, Dako, M0823) diluted in $1 \%$ bovine serum albumin (BSA) in PBS for $45 \mathrm{~min}$ at room temperature. After washing with PBS, cells were incubated with antimouse antibody Alexa Fluor ${ }^{\circledR} 488$ secondary antibody (Life Technologies) diluted $1: 1000$ in $1 \%$ BSA/PBS for $45 \mathrm{~min}$ at room temperature while avoiding light exposure. Additionally, counterstaining of cell nuclei was performed with 4',6-diamidino-2phenylindole (DAPI) $(1: 125)$ and cells were mounted with Fluoroshield (ImmunoBioScience Corp., Mukilteo, WA, USA). Visualisation was carried out using a confocal laser scanning microscope (Leica TCS-NT) and the z-stacks obtained were used for $3 \mathrm{D}$ visualisation using the open source software Image J (Java 1.8.0_112) (Rueden et al., 2017). For immunohistochemistry, samples were fixed in $4 \%$ buffered formalin (Roti-Histofix $4 \%$ acid-free $\mathrm{pH} 7$, Carl-Roth), dehydrated using a tissue processor (Leica TP1020), embedded in paraffin wax, sectioned using a microtome (Leica RM2255) and stained for CD31. Tissue sections were rehydrated in a 
Table 2. Experimental groups. High-RCF or low-RCF PRF were combined with 20,000 cells per well (HFs or ECs) for mono-culture experiments and 10,000 HFs and ECs, respectively, for co-culture experiments. Cells were resuspended in $1 \mathrm{~mL}$ of EBM ${ }^{\mathrm{TM}}-2$ enriched with EGM ${ }^{\mathrm{TM}}-2$ kit supplements.

\begin{tabular}{|c|c|c|c|c|c|c|c|}
\hline & & $\begin{array}{c}\text { Cell culture } \\
\text { medium }\end{array}$ & Collagen & PRF high & PRF low & ECs & HFs \\
\hline \multirow{5}{*}{ Control } & PRF high RCF & $1 \mathrm{~mL}$ & - & $1 \mathrm{~mL}$ & $1 \mathrm{~mL}$ & - & - \\
\hline & PRF low RCF & $1 \mathrm{~mL}$ & - & $1 \mathrm{~mL}$ & $1 \mathrm{~mL}$ & - & - \\
\hline & ECs & $1 \mathrm{~mL}$ & $1 \mathrm{~mL}$ & - & - & - & 20,000 \\
\hline & HFs & $1 \mathrm{~mL}$ & $1 \mathrm{~mL}$ & - & - & 20,000 & - \\
\hline & Co-culture & $1 \mathrm{~mL}$ & $1 \mathrm{~mL}$ & - & - & 10,000 & 10,000 \\
\hline \multirow{3}{*}{ PRF high } & ECs + PRF high & $1 \mathrm{~mL}$ & - & $1 \mathrm{~mL}$ & - & - & 20,000 \\
\hline & HFs + PRF high & $1 \mathrm{~mL}$ & - & $1 \mathrm{~mL}$ & - & 20,000 & - \\
\hline & HFs + ECs + PRF high & $1 \mathrm{~mL}$ & - & $1 \mathrm{~mL}$ & - & 10,000 & 10,000 \\
\hline \multirow{3}{*}{ PRF low } & ECs + PRF low & $1 \mathrm{~mL}$ & - & - & $1 \mathrm{~mL}$ & - & 20,000 \\
\hline & HFs + PRF low & $1 \mathrm{~mL}$ & - & - & $1 \mathrm{~mL}$ & 20,000 & - \\
\hline & HFs+ ECs + PRF low & $1 \mathrm{~mL}$ & - & - & $1 \mathrm{~mL}$ & 10,000 & 10,000 \\
\hline
\end{tabular}

descending ethanol series before commencing the immunostaining protocol. The antigen was retrieved using the heat-induced epitope retrieval (HIER) method (Udeabor et al., 2017) with citrate $\mathrm{pH} 6$ buffer at $95^{\circ} \mathrm{C}$ for $20 \mathrm{~min}$. Samples were rinsed with buffer solution [tris-buffered saline (TBS) with Tween 20 in a ratio of $20: 1]$ before staining with monoclonal mouse anti-human CD31 antibody (1 : 120; Dako, M0823) by Autostainer (Lab Vision Autostainer 360, ThermoFisher Scientific). An anti-mouse secondary antibody was used (HRP Ultravision Quanto Detection System, ThermoFisher Scientific) and chromogenic visualisation was achieved using AEC peroxidase (Dako). Histological examination was performed using a light microscope (Nikon Eclipse 80i) and images were taken with a connected Nikon DS-Fi1/digital camera and a Nikon digital sight unit DS-L2.

\section{Measurements of vascular structures}

Immunohistochemical staining analyses were conducted using an established protocol (Dohle et al., 2017). A 20× magnification scan of each sample was obtained using a light microscope (Nikon Eclipse $\left.80 \mathrm{i}^{\circledR}\right)$ equipped with an automatic scanning table (ProScan III, Prior, Rockland, MA, USA), a Nikon DS-Fi1 digital camera and a Nikon Digital sight DS-L2 unit. The histochemically stained structures (CD31) were manually selected using the area tool of the "annotations and measurements" function of the NIS Elements software (Nikon). The percent vascularisation was calculated as the total area of the vessel/lumen structures divided by the total area of the image. For image quantification, three images for each experimental group were analysed.

\section{Statistical analyses}

Results were calculated as mean \pm standard deviation (SD) and evaluated for significant differences at each time point with one-way analysis of variance (ANOVA) using GraphPad Prism6.0 software (GraphPad Software Inc.). Differences were considered statistically significant for ${ }^{*} p<0.05$, ${ }^{* *} p<0.01,{ }^{* * *} p<0.001$ and ${ }^{* * * *} p<0.0001$. The compiled data were plotted with GraphPad Prism 6.0 software.

\section{Results}

Effects of low- and high-RCF PRF on angiogenic structure formation

Cell/PRF-complexes (ECs + PRF and ECs + HFs + PRF using low-RCF and high-RCF PRF), as well as the control groups without addition of PRF, were stained for immunofluorescence detection of the EC-specific marker CD31 to identify ECs (Fig. 1,2). Additionally, cell/PRF-complexes were stained for immunohistochemical detection of CD31 to visualise vessel-like luminal structures (Fig. 3). Qualitative 
evaluation of CD31 immunofluorescence in the control group without PRF (ECs) revealed a typical cobblestone-like morphology of ECs in mono-culture, which were organised into a monolayer after 7 and $14 \mathrm{~d}$ of cultivation (Fig. 1a,g). ECs in control cocultures consisting of ECs and HFs without addition of PRF were organised into EC aggregates after $7 \mathrm{~d}$ of cultivation (Fig. 1d) and started to form few angiogenic structures after $14 \mathrm{~d}$ of co-cultivation (Fig. 1j). In contrast, combining ECs in mono-culture with PRF generally resulted in a distinct angiogenic activation of ECs, suggested by the formation of many microvessel-like structures (Fig. 1b,c,h,i) after 7 and $14 \mathrm{~d}$, compared to the control groups without the addition of PRF. This PRF-mediated effect on the induction of angiogenesis was even more clear when low-RCF PRF was applied (Fig. 1c,i). This difference in the angiogenic activation between the two PRF preparations was distinct after both cultivation time points. When ECs were combined with lowRCF PRF, ECs formed a very complex network of microvessel-like structures with larger lumena invading the PRF matrix (Fig. 2). Although the formation of single microvessel-like structures was detected when EC/HF co-cultures were combined with PRF, the significant pro-angiogenic effect of PRF on ECs in mono-culture was not observed for the co-cultures (Fig. 1e,f,k,1). The dependence of angiogenic structure formation in ECs on the RCF was also confirmed by immunohistochemistry (Fig.
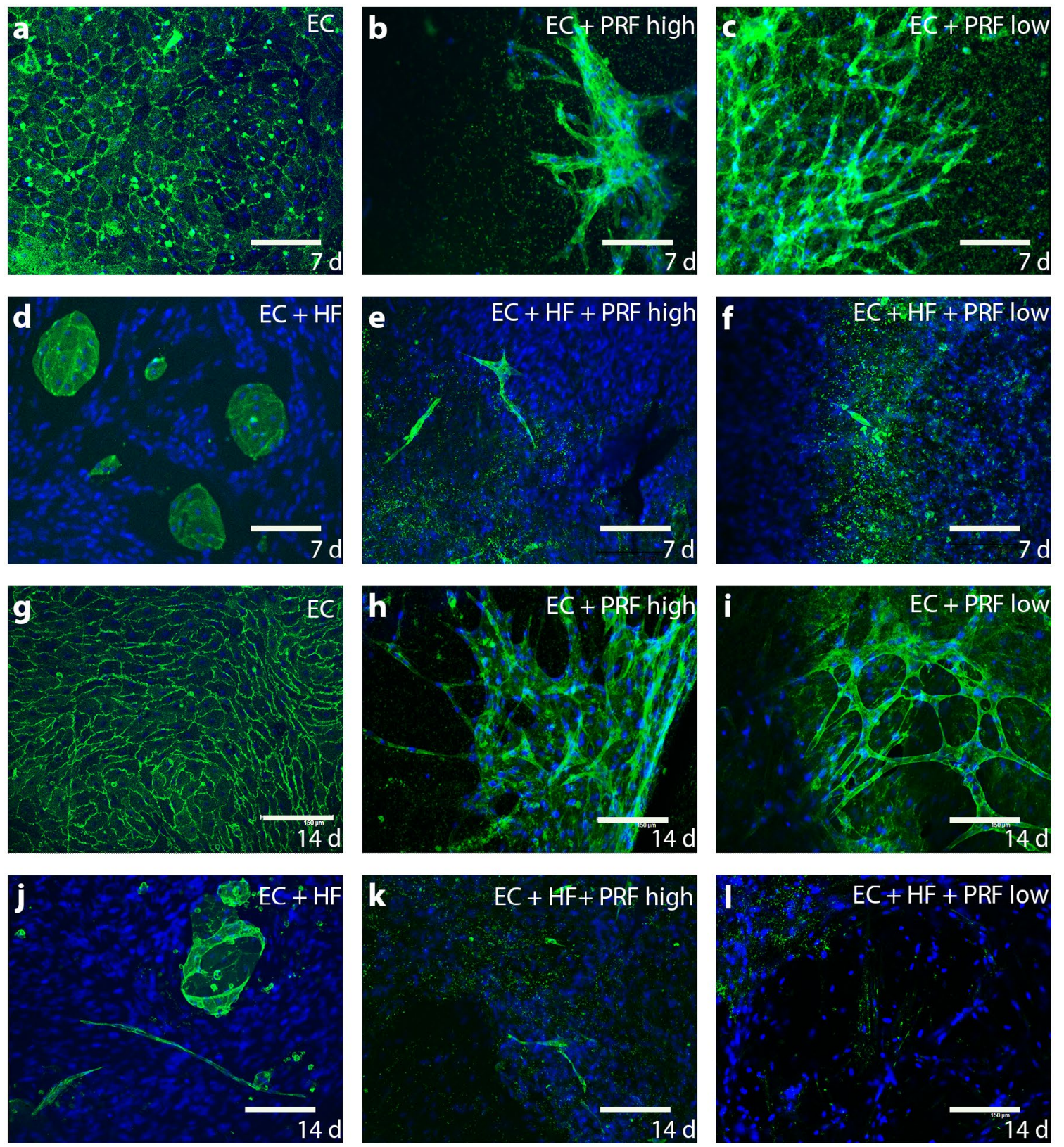

Fig. 1. Lumen and microvessel-like structure formation of ECs in mono-culture and ECs and HFs in coculture mixed with PRF prepared using the LSCC. Cells were cultivated with a (b,e,h,k) high or (c/f/i/l) low RCF PRF as experimental groups and $(\mathbf{a} / \mathbf{d} / \mathbf{g} / \mathbf{j})$ without PRF as control. Whole cell complexes were stained by immunofluorescence for CD31 (green) and DAPI (blue) after (a-f) 7 and (g-l) $14 \mathrm{~d}$ of cultivation. Scale bars: $150 \mu \mathrm{m}$. 
3). CD31 immunostaining of low- and high-RCF PRF complexes with EC and EC/HF co-culture showed a significantly larger amount of CD31-positive luminal structure formation in EC monoculture in which lowRCF PRF had been applied as compared to high-RCF PRF after 7 or $14 \mathrm{~d}$ (Fig. 3). Quantitative evaluation of the total amount of vessel lumina (\% of total area) indicated a statistically larger number of vascular lumina in EC + PRF low-RCF complexes on day 7 and 14 as compared to EC + PRF high-RCF complexes (Fig. 3i). When compared with the immunofluorescence staining of cell/PRF complexes depicted in Fig. 1, co-culture/PRF complexes generally formed vascular luminal structures to a lesser extent on day 7 and 14, although a pro-angiogenic effect of low-RCF PRF was observed for co-culture experiments after $14 \mathrm{~d}$ of cultivation (Fig. 3j).

\section{PRF-mediated effect on protein release in monocultures and co-cultures}

To gain insight into the molecular basis of the pro-angiogenic effect of PRF generated by LSCC, the release of VEGF, PDGF-BB and TGF- $\beta 1$ was measured in all experimental groups after 4, 7 and $14 \mathrm{~d}$ of cultivation (Fig. 4-6). Data compared the effect of PRF on the individual cell types by time points and, more specifically, on ECs and HFs in mono-culture and co-culture relatively to the control (low-RCF PRF without cells) (Fig. 4-6a,b,c). Absolute growth factor concentrations at all time points for the different experimental groups are shown to evaluate growth factor secretion from 4 to $14 \mathrm{~d}$ (Fig. $6 \mathbf{d}, \mathbf{e}, \mathbf{f})$.

\section{VEGF}

On day 4, the highest VEGF concentration was observed in supernatants of HF/low-RCF PRF clots, but this was not statistically significant when compared to the HF + PRF high-RCF and to the HF control group without PRF (Fig. 4a). Although there seemed to be more VEGF in EC supernatants as well as in co-culture supernatants when cells where embedded in low-RCF PRF, compared to the VEGF concentration in supernatants of cells embedded in high-RCF PRF, this result was not statistically significant (Fig. 4a). On day 7, there was an apparent, albeit not statistically significant, increase in VEGF concentration of HFs cultivated with low- or high-RCF PRF as compared to control HFs without PRF (Fig. 4b). In general, there were no distinct differences in VEGF concentration between the supernatants of all other experimental groups after $7 \mathrm{~d}$ of cultivation (Fig. $4 \mathrm{~b}$ ). After $14 \mathrm{~d}$ of cultivation, VEGF content was generally low in supernatants of all tested experimental groups as compared to control HF mono-culture and control low-RCF PRF without additional cells (Fig. 4c). On day 14, VEGF concentration was significantly higher in supernatants of low-RCF PRF as compared to highRCF PRF. The effect of PRF on HFs was clear when the absolute VEGF concentration was evaluated by time point and experimental group (Fig. 4d). VEGF concentration increased in cell culture supernatants when HFs were embedded in PRF with low-RCF and high-RCF PRF from 4 up to $7 \mathrm{~d}$ as compared to control HFs without PRF cultivation. No increase in VEGF protein secretion was measured for ECs in mono-culture or co-culture (Fig. 4e,f).

\section{PDGF-BB}

After the first time point ( $4 \mathrm{~d}$ ), the concentration of PDGF-BB was significantly higher in the supernatants of HF mono-culture, EC mono-culture and co-cultures of ECs and HFs when the cells were combined with low-RCF PRF as compared to the mono-cultures
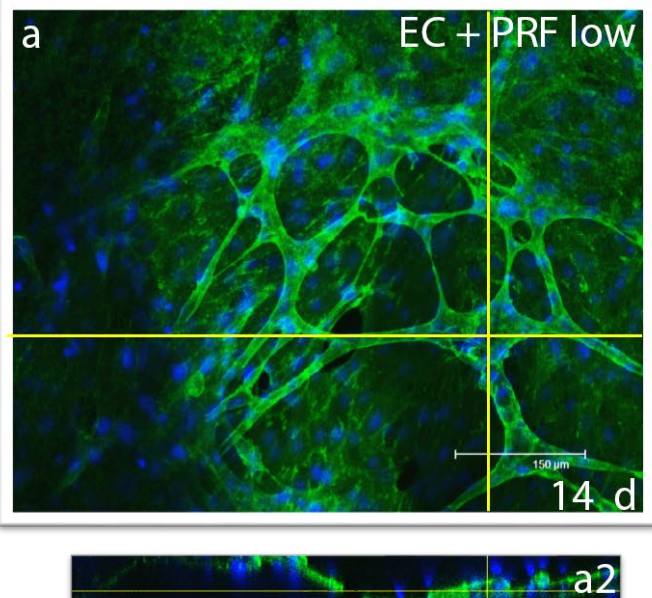

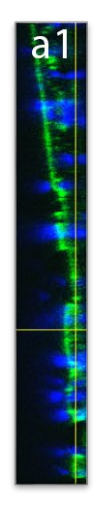

a2
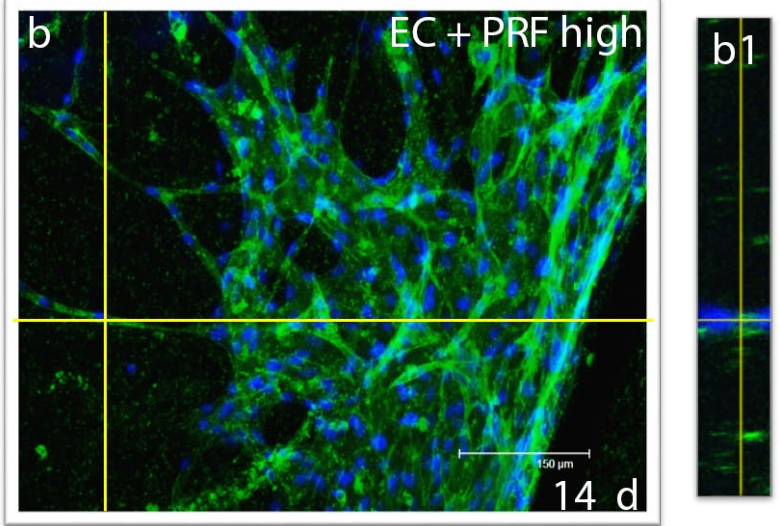

Fig. 2. Immunofluorescence staining of PRF/cell complex after $14 \mathrm{~d}$ of cultivation. Representative images of 3D projections using z-stacks obtained from the confocal microscopy with orthogonal views using the open source software Image J. Qualitative evaluation of lumen size formation by ECs cultivated with (a) PRF low-RCF as compared to (b) PRF high-RCF. The intersection of the yellow lines indicates the centre of the lumen displayed in the YZ (Fig. a1-b1) and XZ (Fig. a2-b2) planes. A more complex network of vessel-like structures with bigger lumen size can be observed in the EC + PRF-low as compared to the EC + PRF-high RCF. Scale bars: $150 \mu \mathrm{m}$. a and $\mathbf{b}$ correspond to Fig. $1 \mathbf{i}$ and $1 \mathbf{h}$, respectively. 
combined with high-RCF PRF or without PRF (Fig. 5a). Similar results were also observed after 7 d (Fig. 5b). PDGF concentration was increased in HF/low-RCF PRF complexes and in EC/lowRCF PRF complexes after $7 \mathrm{~d}$ as compared to the other experimental groups, although statistical significance was found only for low-RCF PRF on EC mono-culture (Fig. 5b). The effect of low-RCF PRF became even clearer at the third time point (Fig. $5 c)$. After $14 \mathrm{~d}$ of cultivation, the concentration of PDGF in supernatants of EC mono-culture increased tremendously in a PRF-dependent manner as compared to control mono-culture and to all other experimental groups. Supernatants of EC/low-RCF PRF complexes contained $\sim 13$ times more PDGF than the corresponding control group without PRF, a result that was statistically significant (Fig. 5c). Additionally, PDGF content in supernatants of ECs combined with high-RCF PRF also increased significantly when compared to control EC mono-cultures. The difference in absolute PDGF concentration between EC supernatants with or without PRF also supported these data (Fig. 5e). The highest PDGF concentration was observed with ECs combined with low-RCF PRF for $14 \mathrm{~d}$, whereas this effect was not observed when HF mono-culture or in co-culture with ECs were combined with PRF (Fig. 5d,f).

\section{TGF- $\beta 1$}

Low-RCF PRF clots without additional external cells released more TGF- $\beta 1$ into the surrounding medium than high-RCF PRF clots at all three time points, with statistical significance after 4 and $7 \mathrm{~d}$ of cultivation (Fig. 6a,b,c). After 4 and 7 d, TGF- $\beta 1$ concentration in cell culture supernatants was significantly higher than in supernatants of HFs and ECs in mono-culture and co-culture when the cells were mixed with lowRCF PRF as compared to control cells without PRF and cells embedded in high-RCF PRF (Fig. 6a,b). After $14 \mathrm{~d}$, there were no distinct differences between the different experimental groups in terms of detectable TGF- $\beta 1$ (Fig. 6c). Although TGF- $\beta 1$ concentration in the cell culture supernatants of ECs, HFs and cocultures was very high with low-RCF PRF at $4 \mathrm{~d}$, it gradually decreased to a level comparable to that in supernatants of high RCF PRF clots with monocultures and supernatants of cells without PRF after $14 \mathrm{~d}$ of cultivation (Fig. $6 \mathrm{~d}, \mathbf{e}, \mathbf{f}$ ).

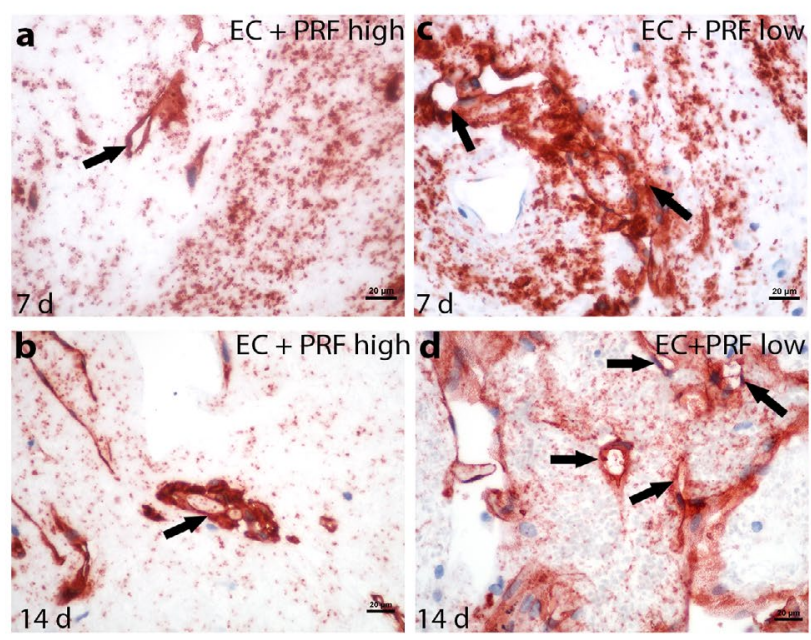

i

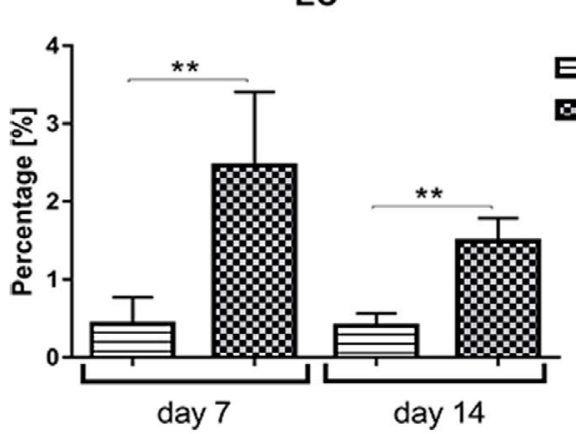

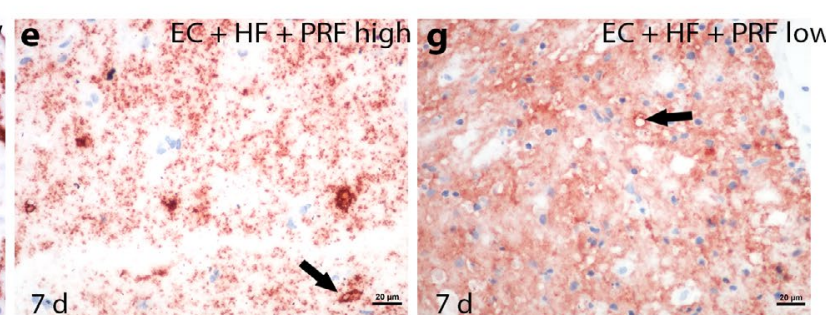

f. $\mathrm{EC}+\mathrm{HF}+\mathrm{PRF}$ high h $\mathrm{h}$.

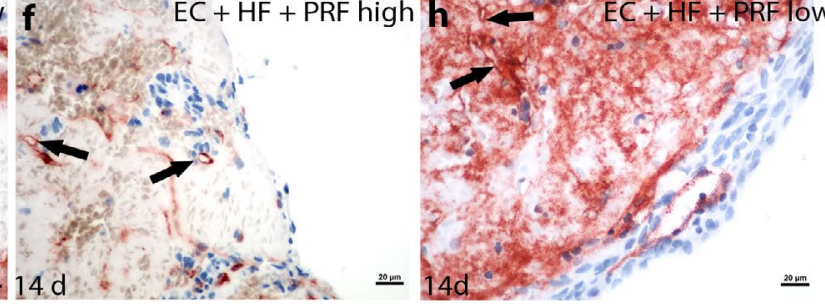

j

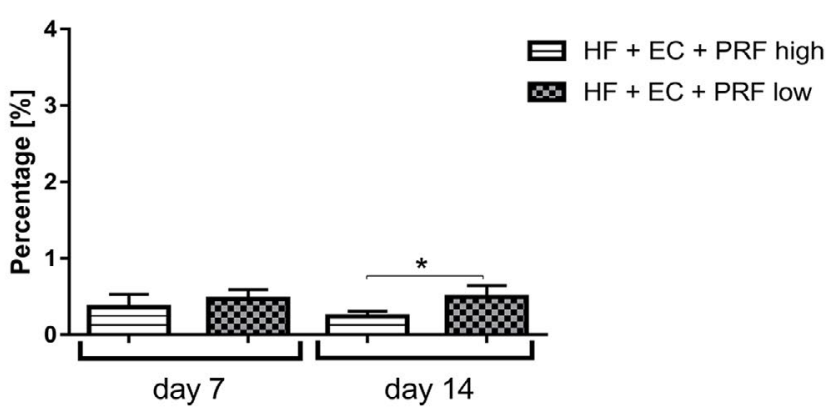

Fig. 3. Lumen and microvessel-like structure formation of ECs in mono-culture and ECs and HFs in cocultures mixed with PRF prepared using the LSCC. Cells were cultivated with a $(\mathbf{a}, \mathbf{b}, \mathbf{e}, \mathbf{f})$ high- or $(\mathbf{c}, \mathbf{d}, \mathbf{g}, \mathbf{h})$ low-RCF PRF. Histological sections of PRF-cell complexes were stained immunohistochemically for CD31 after 7 and $14 \mathrm{~d}$ of cultivation. Black arrows highlight positively stained lumen structures formation. (a-h) Scale bars: $20 \mu \mathrm{m}$. $(\mathbf{i}, \mathbf{j})$ The percentage (\%) of vascularisation was calculated from the total vessel/lumen area and the total area of the images. Data error bars are calculated as SD of the mean. Statistical significance was evaluated by means of ANOVA. Differences were considered statistically significant for ${ }^{*} p<0.05$ and ** $p<0.01$. 


\section{Discussion}

The use of PRF as a promising material for tissue engineering has recently gained attention among dentists in the field of regenerative medicine since PRF seems to promote a material-induced physiological tissue reaction in defective areas that leads to better wound healing and tissue repair (Miron et al., 2017). PRF is a blood concentrate containing inflammatory cells and growth factors embedded in a fibrin network, which can be easily obtained from a patient's own peripheral blood using a one-step centrifugation process without addition of anticoagulants (Choukroun, 2001; Ghanaati et al., 2014). With regard to this, the cellular and structural composition of PRF can be modified through the application of different RCFs and centrifugation times (Choukroun and Ghanaati, 2018; El Bagdadi et al., 2017). Implementation of the LSCC resulted in an increased concentration of inflammatory cells and growth factors released from injectable and solid PRF matrices as a consequence of a reduction in the RCF (El Bagdadi et al., 2017; Wend et al., 2017). Wend et al. (2017) showed that decreasing the RCF leads to a larger number of leukocytes, lymphocytes, neutrophils, monocytes and platelets in the resulting injectable PRF when compared to PRF generated at higher RCFs. Therefore, the amount of growth factor and cytokine release in PRF matrices increases with decreasing RCF forces. The present study investigated, for the first time, two different PRF matrices produced at low- $(44 \times g)$ and high$(710 \times g)$ RCF and their influence on the formation of angiogenic structures and growth factor release, using HDMECs in mono-culture and in co-culture with HFs as an in vitro model system for soft tissue regeneration. In general, the present comparative study revealed a distinct PRF-dependent, proangiogenic effect on ECs in mono-culture, as demonstrated by an increase in microvessel-like structures when ECs where combined with both types of PRF as compared to the appropriate control groups without PRF. Interestingly, this pro-angiogenic effect of PRF on ECs was significantly larger when low- a

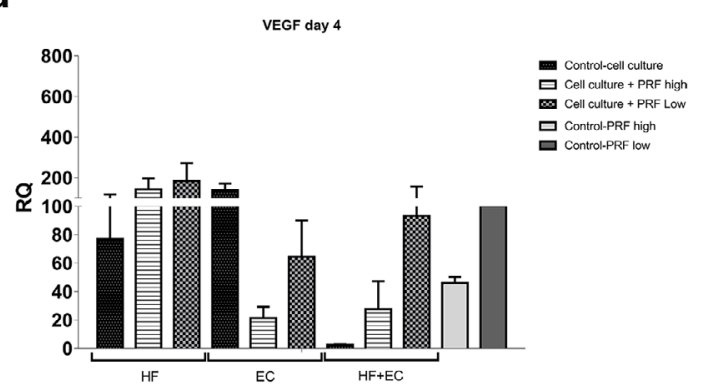

b

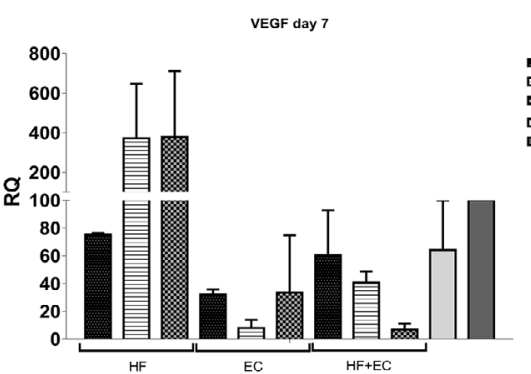

C

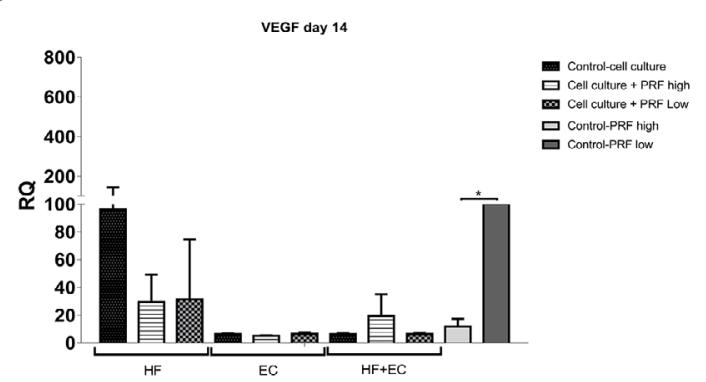

d
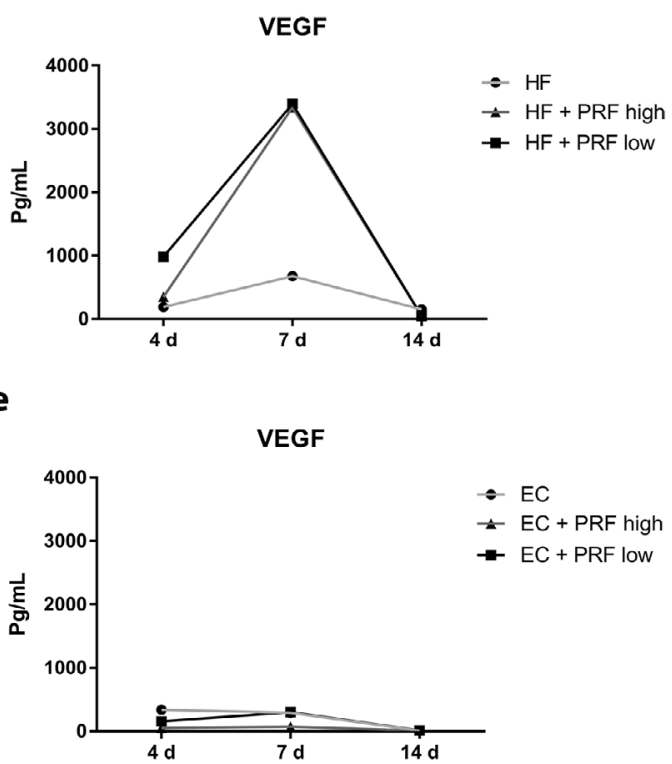

f

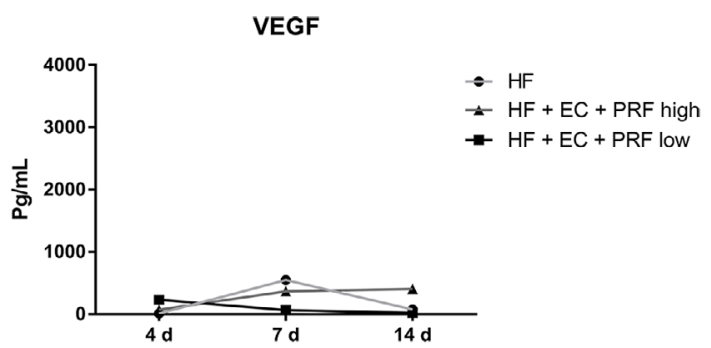

Fig. 4. Quantification of VEGF content within the cell culture supernatants released from cell/PRFcomplexes by ELISA. Mono- and co-cultures were cultivated with a high or low RCF PRF prepared using the LSCC; control group was cultivated without PRF. (a-c) Results are relatively quantified (RQ) to the control PRF low-RCF matrix without additional cells. (d-f) Absolute concentrations at 4, 7 and $14 \mathrm{~d}$. Data error bars are calculated as SD of the mean. Statistical significance was evaluated by means of ANOVA. Differences were considered statistically significant for ${ }^{*} p<0.05,{ }^{* *} p<0.01,{ }^{* *} p<0.001$ and ${ }^{* * *} p<0.0001$. 
RCF PRF was employed as compared to high-RCF PRF. Analysis of growth factor production in PRF produced at different RCF confirmed previous results (Choukroun and Ghanaati, 2018; El Bagdadi et al., 2017; Wend et al., 2017). A larger release of TGF- $\beta 1$ and PDGF-BB in low-RCF PRF and in low-RCF PRF combined with cells was observed, indirectly suggesting that larger cell numbers were generated in the low PRF/cell complexes by the LSCC. Considering that reducing the applied RCF in low-RCF PRF results in a higher concentration of leukocytes and their subtypes, especially neutrophils (Ghanaati et al. 2014), the observed pro-angiogenetic effect may be related to the presence of a large number of neutrophils and their released neutrophil extracellular traps (NETs). This is supported by an in vitro study showing the positive influence of neutrophils on angiogenesis and osteogenesis in vitro (Herath et al., 2018). In addition, during tissue repair, activated platelets accumulate in the wound area and form a fibrin clot that closes the wound and secretes growth factors - such as PDGF or TGF- $\beta 1$ - which are, therefore, key players in the activation of immune cells, wound healing and tissue regeneration (Gurtner et al., 2008; Martin, 1997). In particular, the interaction of platelets with leukocytes, such as monocytes, macrophages and neutrophils, is a requisite for normal wound healing and tissue regeneration. Activated immune cells, such as monocytes, release different growth factors, e.g. PDGF-BB and TGF- $\beta 1$, that attract fibroblasts to sites of injury where they produce an extracellular matrix within the wound tissue (Eming et al., 2007; Werner and Grose, 2003). Additionally, TGF- $\beta 1$, PDGF-BB and VEGF in concert are essential for the stabilisation of vascular structures (Beck and D'Amore, 1997). The larger release of PDGF-BB and TGF- $\beta 1$ in the lowRFC PRF/cell complexes as compared to the high-RCF $\mathrm{PRF} /$ cell complexes resembled the initiation of wound a

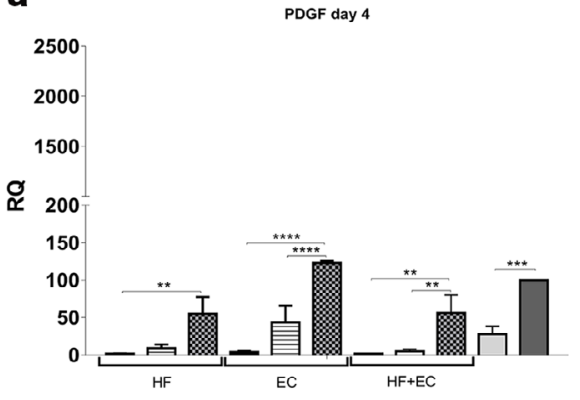

b

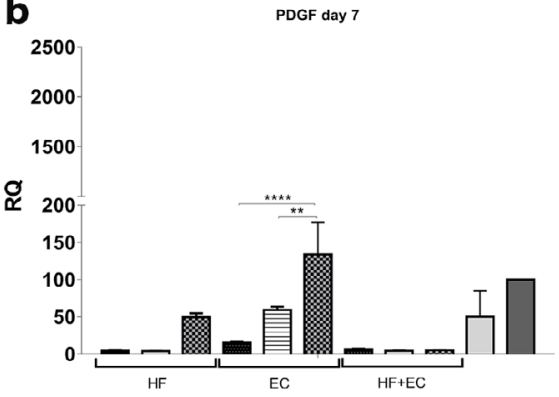

C

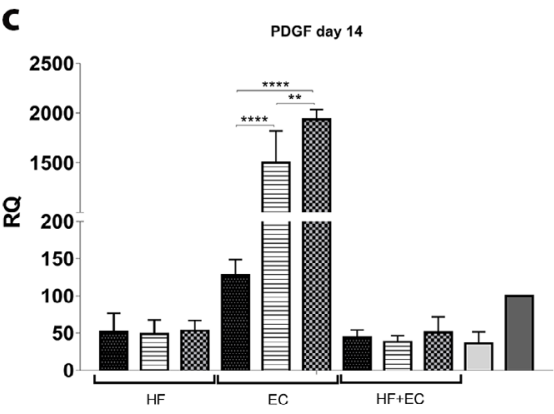

d
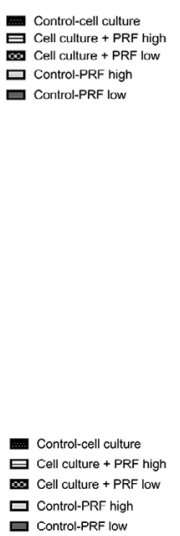

e

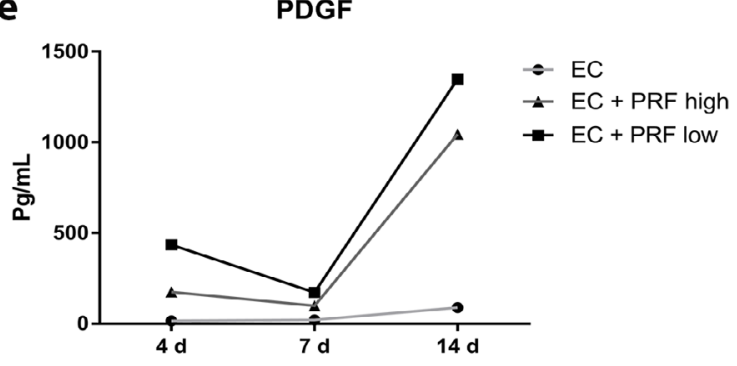

f

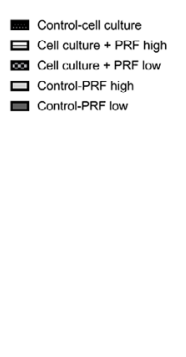

PDGF

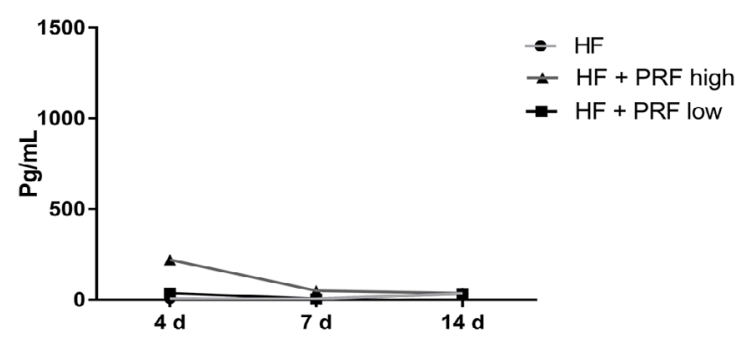

PDGF 口 Control-PRF high

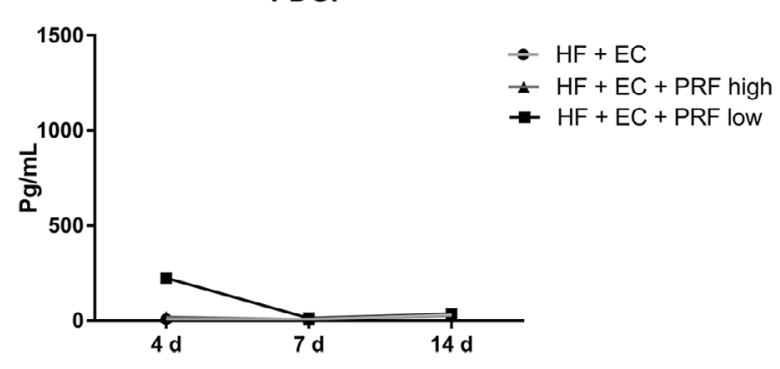

Fig. 5. Quantification of PDGF-BB content within the cell culture supernatants released from cell/PRFcomplexes by ELISA. Mono- and co-cultures were cultivated with a high- or low-RCF PRF prepared using the LSCC; control group was cultivated without PRF. (a-c) Results are relatively quantified (RQ) to the control PRF low-RCF matrix without additional cells. (d-f) Absolute concentrations at 4, 7 and $14 \mathrm{~d}$. Data error bars are calculated as SD of the mean. Statistical significance was evaluated by means of ANOVA. Differences were considered statistically significant for ${ }^{*} p<0.05,{ }^{* *} p<0.01,{ }^{* * *} p<0.001$ and ${ }^{* * *} p<0.0001$. 
healing mediated by the addition of PRF produced by the LSCC. Furthermore, activated leukocytes secrete VEGF within the wound area, which finally leads to the induction of blood vessel formation, underscoring the close interaction of the different cell types during wound healing processes and angiogenesis (Jenne et al., 2013; Nurden, 2011).

The results obtained could provide an idea about the potential origin of the growth factor and their pattern of consumption or accumulation in the cell culture supernatant, leading to vascular formation. The significant pro-angiogenic effect of low-RCF PRF on ECs may be attributed to higher VEGF expression, since the release of VEGF was observed to be larger in low-RCF PRF as compared to high-RCF PRF, leading to the assumption that this difference in VEGF expression was already triggering the ECs to form microvessel-like structures. Given

a
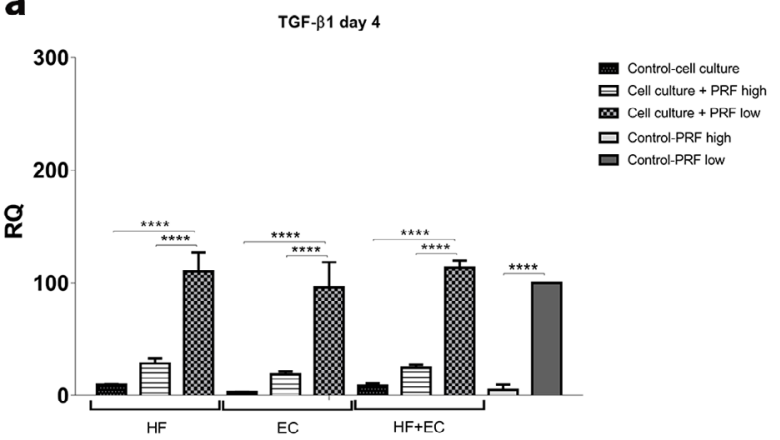

b

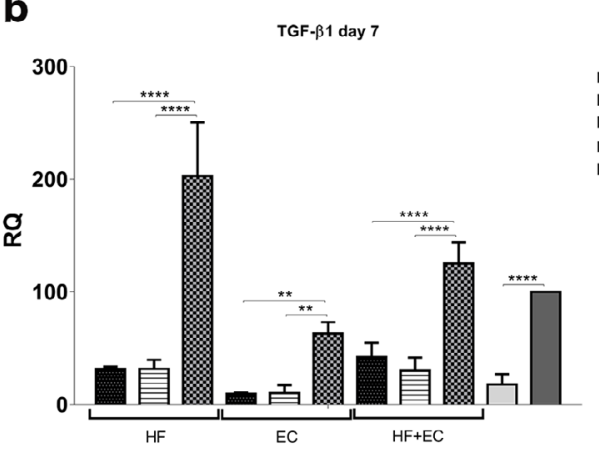

C
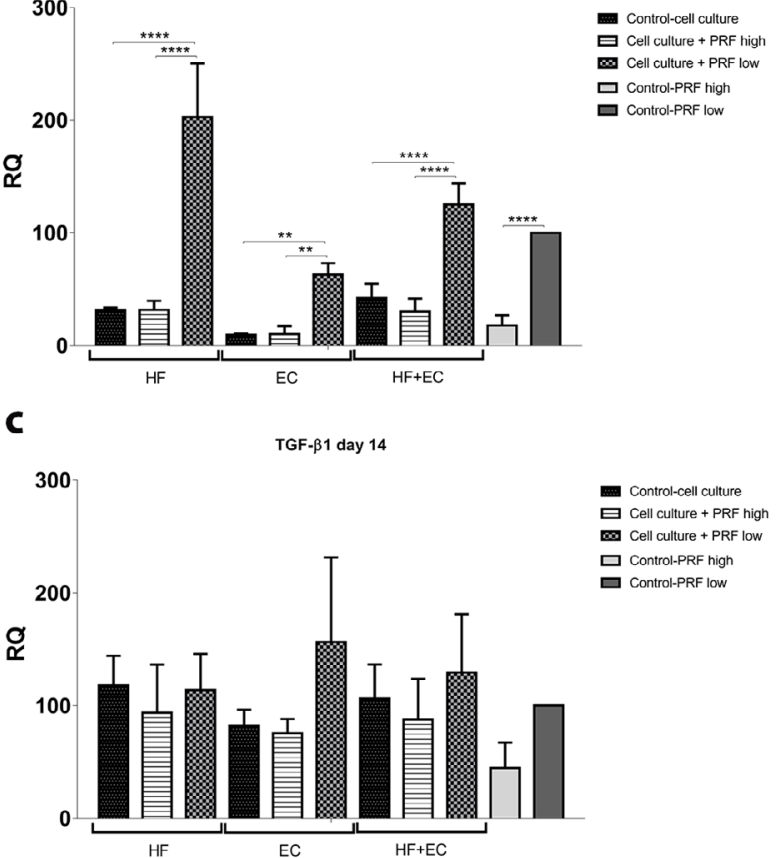

that there was almost no detectable VEGF in the EC/PRF groups after $14 \mathrm{~d}$ of cultivation, it is reasonable to assume that during cultivation with PRF, ECs adsorbed the free VEGF released from PRF into the supernatants by VEGF binding to its receptor, VEGF receptor 2 (VEGFR2). This led to their angiogenic activation, which finally resulted in microvessel-like structure formation. Blocking of soluble VEGF using conditioned medium from soluble VEGFR2-producing cells results in VEGF ligand inhibition, reduction of free VEGF in cell culture supernatants and inhibition of angiogenesis in a triple-culture model system for bone tissue engineering (Dohle et al., 2014). In contrast, the lower observed concentrations of growth factors and angiogenic structure formation in the PRF/co-culture complexes as compared to the mono-culture might be associated with an excessive supply of growth factors,

d

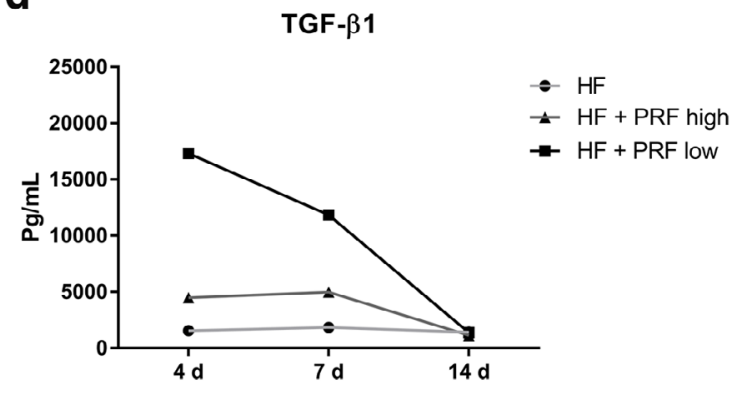

e

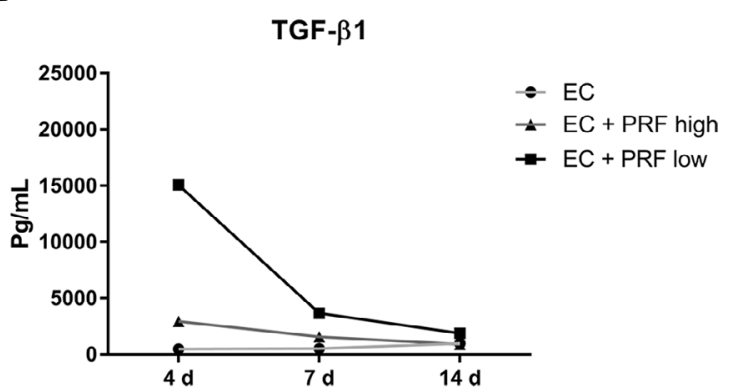

f

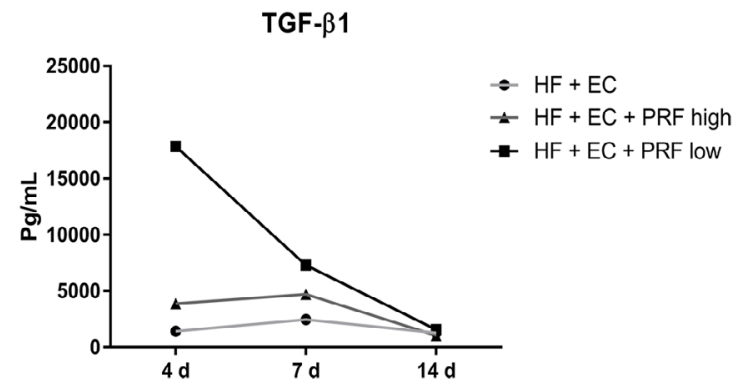

Fig. 6. Quantification of TGF- $\beta 1$ content within the cell culture supernatants released from cell/PRFcomplexes by ELISA. Mono- and co-cultures were cultivated with a high- or low-RCF PRF prepared using the LSCC; control group was cultivated without PRF. (a-c) Results are relatively quantified (RQ) to the control PRF low-RCF matrix without additional cells. (d-f) Absolute concentrations at 4, 7 and $14 \mathrm{~d}$. Data error bars are calculated as SD of the mean. Statistical significance was evaluated by means of ANOVA. Differences were considered statistically significant for ${ }^{*} p<0.05,{ }^{* *} p<0.01,{ }^{* * *} p<0.001$ and ${ }^{* * * *} p<0.0001$. 
i.e. VEGF and TGF- $\beta 1$, deriving from PRF itself and additionally released by HFs. In general, highest VEGF and TGF- $\beta 1$ concentrations could be found in HF/PRF-complexes after 4 and $7 \mathrm{~d}$. Growth factor concentrations in supernatants of co-culture/PRF complexes were comparatively lower, supposedly being consumed by ECs in these complexes. Both VEGF and TGF- $\beta 1$ might induce angiogenesis but have opposite effects on ECs, since the crosstalk between these growth factors also controls ECs apoptosis (Hicklin and Ellis, 2005). VEGF protects ECs from apoptosis whereas TGF- $\beta 1$ induces apoptosis, demonstrating the necessity for a precise regulation of their expression and interaction (Ferrari et al., 2006; Ferrari et al., 2009) In addition, TGF- $\beta 1$ downregulates VEGFR2 expression, therefore enabling abolishment of angiogenic structure formation mediated through VEGF (Mandriota et al., 1996) and, possibly, limiting VEGF effect in the co-culture/PRF-complexes.

Another explanation for the PRF-mediated proangiogenic effect in this in vitro model relates to the fibrin structure of PRF itself, which serves as a 3D matrix. In vasculogenesis models, the $3 \mathrm{D}$ matrix is a sine qua non condition for the formation of $3 \mathrm{D}$ vascular structures in vitro (Vailhe et al., 2001). In terms of cellbased vascularisation strategies, the rapid initiation of neovascularisation in vivo can only be achieved by co-implantation of pro-angiogenic matrices such as Matrige $\mathrm{l}^{\circledR}$ (Fuchs et al., 2009). In previous in vitro 3D culture models, the modification of the mechanical properties (i.e. Young's modulus, stress/strain) of synthetic manufactured extracellular matrices (S-ECM) originating from collagen, hyaluronic acid or fibrin hydrogel has shown an effect on cellular physiological processes (Nebuloni et al., 2016; Soofi et al., 2009). Based on previous studies, it is arguable that the structure of PRF could be modified by the RCF (Kubesch et al. 2018). In this context, more vascularisation could be expected in vivo in nude mice, depending on the porous structure of the implanted PRF. The changes in fibrin structure gained from different RCFs could be pulled together with a larger number of angiogenic structures in the more porous low-RCF PRF matrix as compared to the highRCF PRF and control group. The effect of the fibrin structure of PRF on the angiogenic arrangement of ECs is documented in vivo (Kubesch et al. 2018). The tissue reaction in subcutaneous implantation model of severe combined immunodeficient (SCID) mice clearly differs from the present in vivo results, likely due to differences in the implanted fibrin scaffold composition and porous structure of the implanted PRF matrix. According to Kubesch et al. (2018), the implanted low-RCF PRF has a more porous structure and results in a higher vascularisation rate as compared to implantation of high-RCF PRF. In general, a more porous and softer matrix appears to facilitate angiogenesis. Therefore, fine-tuning the mechanical properties of PRF matrices for in vitro cell tissue engineering and for clinical practices (i.e. plug
PRF, pressed or unpressed PRF matrices) to resemble the cell microenvironment should be a topic of further studies.

The aim of the present study was to compare the distinct proangiogenic effect of low- and highRCF PRF with regard to EC angiogenic activation. The applied methods contributed to refute that angiogenesis was not being facilitated through cocultivation itself and permitted the observations that angiogenic structures were, in fact, induced by PRF. A limitation of the study may be the absence of a control group using a S-ECM. In this regard, further experimental scenarios with different S-ECM as control groups are currently being conducted to determine if the proangiogenic effect of PRF is mediated by cell content, matrix structure and/or its secretome. Additionally, phenotypic characterisation of ECs and HFs during cell culture was not performed. This led to the question whether a high growth factor concentration is a possible risk factor if PRF is applied clinically, considering the observed proangiogenic effects. To the authors' knowledge, there is no information in the literature regarding side effects or tumorigenesis induced by PRF. In a recently published systematic review involving 73 clinical studies, no reports were found of any side effects or tumorigenesis after PRF application for the treatment of different pathologies (Ghanaati et al., 2018b). Furthermore, long-term clinical follow-up studies can be found in the literature describing no negative effects of PRF application up to 72 months (Simonpieri et al., 2011). Additionally, preliminary results of temporomandibular joint (TMJ) infiltrations with i-PRF for treatment of a pathological state known as "internal derangement" and localised TMJ pain were reported by Albilia et al. (2018). 37 patients participated in the study with an observation period of 12 months. During the study, the patients reported reduction of pain and improvement of function. No side effects were observed and the patients are, currently, still under observation (Albilia et al., 2018). In an additional clinical case, Ghanaati et al. (2018a) reported the use of i-PRF as a filling skin material for treating hyaluronic-acid-related complications, with a follow up of 24 months. In this case, PRF injections promoted wound healing and minimised scar formation with no clinical signs of tumorigenesis induction (Ghanaati et al., 2018a). In a further clinical report, a patient affected by squamous cell carcinoma in the anterior floor of the mouth was subjected to a tumour resection including an anterior block resection of the mandible. After 16 months of tumour therapy, the patient underwent reconstruction with an individualised 3D titanium mesh and the combination of PRF with a xenogeneic bone substitute biomaterial (Bios-Oss ${ }^{\circledR}$, Geistlich Biomaterials, Wolhusen, Switzerland). After a follow up of 12 months, the patient was rehabilitated with dental implants and prosthetics. The histological analyses of bone biopsies showed new bone 
formation and a well vascularised connective tissue. It is noteworthy that after 28 months of follow up there was no evidence of tumour recurrence (Lorenz et al., 2018).

Based on previous studies that evaluated single components of the peripheral blood [i.e. platelets (Nurden 2011), neutrophils (Herath et al., 2018) and monocytes (Barbeck et al., 2016)] to understand their individual role in tissue engineering, the present study proposed a complex system containing cells and proteins existing within the peripheral blood and aimed at understanding their effect at different concentrations, using high- and low-RCF to vary their amounts. The important role of the secretome for tissue regeneration has come into focus, as it was found to contain the necessary chemokines, cytokines and growth factors that positively affect wound healing (Burdon et al., 2011; Gaetani et al., 2018). Therefore, PRF might be a useful therapeutic "biologisation" instrument of acellular biomaterials to increase their regenerative potential by providing additional secretion of growth factors with increased pro-angiogenic activity. Reducing the RCF of PRF had a significant influence on growth factor secretion and induction of angiogenesis in an in vitro model system for soft tissue engineering in which immune cells, growth factors and the structure of the fibrin matrix seemed to act together to create an appropriate microenvironment that might improve wound healing and vascularisation.

\section{Conclusion}

The present study evaluated the effect of PRF produced either with a high- $(710 \times g)$ or low- $(44 \times g)$ RCF according to the LSCC on human vascular ECs and HFs in mono-culture and in co-culture. PRFmediated effects on the growth factor performance and the pro-angiogenic effect of PRF on this in vitro model system were analysed. PRF generated with low-RCF and combined with ECs resulted in a significantly larger release of growth factors and a significantly larger number of microvessel-like structures as compared to high-RCF PRF and control groups. In conclusion, PRF is a beneficial tool for increasing growth factor release and angiogenesis for in vitro cell tissue-engineering applications. Using low-RCF PRF, most likely meeting good clinical practice guidelines, it might be possible to facilitate a material-induced physiological tissue reaction in defective areas, leading to better wound healing and tissue repair.

\section{Acknowledgements}

The authors declare no conflict of interest and no competing financial interests exist. This work was funded by the FORM-lab. J. Choukroun is the owner of Process for PRF, Nice, France.

\section{References}

Al-Maawi S, Orlowska A, Sader R, James Kirkpatrick C, Ghanaati S (2017) In vivo cellular reactions to different biomaterials-physiological and pathological aspects and their consequences. Semin Immunol 29: 49-61.

Albilia JB, Weisleder H, Wolford LM (2018) Treatment of posterior dislocation of the mandibular condyle with the double mitek mini anchor technique: a case report. J Oral Maxillofac Surg 76: 396.e1-396. e9. DOI: 10.1016/j.joms.2017.09.017.

Auger FA, Gibot L, Lacroix D (2013) The pivotal role of vascularization in tissue engineering. Annu Rev Biomed Eng 15: 177-200.

Barbeck M, Najman S, Stojanovic S, Mitic Z, Zivkovic JM, Choukroun J, Kovacevic P, Sader R, Kirkpatrick CJ, Ghanaati S (2015) Addition of blood to a phycogenic bone substitute leads to increased in vivo vascularization. Biomed Mater 10: 055007. DOI: 10.1088/1748-6041/10/5/055007.

Barbeck M, Unger RE, Booms P, Dohle E, Sader RA, Kirkpatrick CJ, Ghanaati S (2016) Monocyte preseeding leads to an increased implant bed vascularization of biphasic calcium phosphate bone substitutes via vessel maturation. J Biomed Mater Res A 104: 2928-2935.

Beck L, Jr., D’Amore PA (1997) Vascular development: cellular and molecular regulation. FASEB J 11: 365-373.

Burdon TJ, Paul A, Noiseux N, Prakash S, Shum-Tim D (2011) Bone marrow stem cell derived paracrine factors for regenerative medicine: current perspectives and therapeutic potential. Bone Marrow Res 2011: 207326. DOI: 10.1155/2011/207326.

Choukroun J, Diss A, Simonpieri A, Girard MO, Schoeffler C, Dohan SL, Dohan AJ, Mouhyi J, Dohan DM (2006a) Platelet-rich fibrin (PRF): a secondgeneration platelet concentrate. Part IV: clinical effects on tissue healing. Oral Surg Oral Med Oral Pathol Oral Radiol Endod 101: e56-60.

Choukroun J, Diss A, Simonpieri A, Girard MO, Schoeffler C, Dohan SL, Dohan AJ, Mouhyi J, Dohan DM (2006b) Platelet-rich fibrin (PRF): a second-generation platelet concentrate. Part V: histologic evaluations of PRF effects on bone allograft maturation in sinus lift. Oral Surg Oral Med Oral Pathol Oral Radiol Endod 101: 299-303.

Choukroun J, Ghanaati S (2018) Reduction of relative centrifugation force within injectable plateletrich-fibrin (PRF) concentrates advances patients' own inflammatory cells, platelets and growth factors: the first introduction to the low speed centrifugation concept. Eur J Trauma Emerg Surg 44: 87-95.

Choukroun J, Adda F, Schoeffler C, Vervelle A (2001) Une opportunite' en paro-implantologie: le PRF. Implantodontie 42: 55-62.

DeCicco-Skinner KL, Henry GH, Cataisson C, Tabib T, Gwilliam JC, Watson NJ, Bullwinkle EM, Falkenburg L, O'Neill RC, Morin A, Wiest JS (2014) Endothelial cell tube formation assay for the in 
vitro study of angiogenesis. J Vis Exp: e51312. DOI: 10.3791/51312.

Dohan DM, Choukroun J, Diss A, Dohan SL, Dohan AJ, Mouhyi J, Gogly B (2006a) Platelet-rich fibrin (PRF): a second-generation platelet concentrate. Part I: technological concepts and evolution. Oral Surg Oral Med Oral Pathol Oral Radiol Endod 101: e37-44.

Dohan DM, Choukroun J, Diss A, Dohan SL, Dohan AJ, Mouhyi J, Gogly B (2006b) Platelet-rich fibrin (PRF): a second-generation platelet concentrate. Part II: platelet-related biologic features. Oral Surg Oral Med Oral Pathol Oral Radiol Endod 101: e45-50.

Dohan DM, Choukroun J, Diss A, Dohan SL, Dohan AJ, Mouhyi J, Gogly B (2006c) Platelet-rich fibrin (PRF): a second-generation platelet concentrate. Part III: leucocyte activation: a new feature for platelet concentrates? Oral Surg Oral Med Oral Pathol Oral Radiol Endod 101: e51-55.

Dohle E, Bischoff I, Bose T, Marsano A, Banfi A, Unger RE, Kirkpatrick CJ (2014) Macrophagemediated angiogenic activation of outgrowth endothelial cells in co-culture with primary osteoblasts. Eur Cell Mater 27: 149-165.

Dohle E, El Bagdadi K, Sader R, Choukroun J, James Kirkpatrick C, Ghanaati S (2017) Platelet-rich fibrin-based matrices to improve angiogenesis in an in vitro co-culture model for bone tissue engineering. J Tissue Eng Regen Med 12: 598-610.

El Bagdadi K, Kubesch A, Yu X, Al-Maawi S, Orlowska A, Dias A, Booms P, Dohle E, Sader R, Kirkpatrick CJ, Choukroun J, Ghanaati S (2017) Reduction of relative centrifugal forces increases growth factor release within solid platelet-rich-fibrin (PRF)-based matrices: a proof of concept of LSCC (low speed centrifugation concept). Eur J Trauma Emerg Surg. DOI: 10.1007/s00068-017-0785-7.

Eming SA, Krieg T, Davidson JM (2007) Inflammation in wound repair: molecular and cellular mechanisms. J Invest Dermatol 127: 514-525.

Ferrari G, Cook BD, Terushkin V, Pintucci G, Mignatti P (2009) Transforming growth factorbeta 1 (TGF-beta1) induces angiogenesis through vascular endothelial growth factor (VEGF)-mediated apoptosis. J Cell Physiol 219: 449-458.

Ferrari G, Pintucci G, Seghezzi G, Hyman K, Galloway AC, Mignatti P (2006) VEGF, a prosurvival factor, acts in concert with TGF-beta1 to induce endothelial cell apoptosis. Proc Natl Acad Sci U S A 103: 17260-17265.

Fuchs S, Ghanaati S, Orth C, Barbeck M, Kolbe M, Hofmann A, Eblenkamp M, Gomes M, Reis RL, Kirkpatrick CJ (2009) Contribution of outgrowth endothelial cells from human peripheral blood on in vivo vascularization of bone tissue engineered constructs based on starch polycaprolactone scaffolds. Biomaterials 30: 526-534.

Gaetani M, Chinnici CM, Carreca AP, Di Pasquale C, Amico G, Conaldi PG (2018) Unbiased and quantitative proteomics reveals highly increased angiogenesis induction by the secretome of mesenchymal stromal cells isolated from fetal rather than adult skin. J Tissue Eng Regen Med 12: e949-e961.

Ghanaati S, Al-Maawi S, Schaffner Y, Sader R, Choukroun J, Nacopoulos C (2018a) Application of liquid platelet-rich fibrin for treating hyaluronic acidrelated complications: a case report with 2 years of follow-up. Int J Growth Factors Stem Cells Dent 1: 74-77.

Ghanaati S, Booms P, Orlowska A, Kubesch A, Lorenz J, Rutkowski J, Landes C, Sader R, Kirkpatrick C, Choukroun J (2014) Advanced platelet-rich fibrin: a new concept for cell-based tissue engineering by means of inflammatory cells. J Oral Implantol 40: 679-689.

Ghanaati S, Fuchs S, Webber MJ, Orth C, Barbeck M, Gomes ME, Reis RL, Kirkpatrick CJ (2011) Rapid vascularization of starch-poly(caprolactone) in vivo by outgrowth endothelial cells in co-culture with primary osteoblasts. J Tissue Eng Regen Med 5: e136143.

Ghanaati S, Herrera-Vizcaino C, Al-Maawi S, Lorenz J, Miron RJ, Nelson K, Schwarz F, Choukroun J, Sader R (2018b) Fifteen years of platelet rich fibrin (PRF) in dentistry and oromaxillofacial surgery: how high is the level of scientific evidence? J Oral Implantol 44: 471-492.

Gurtner GC, Werner S, Barrandon Y, Longaker MT (2008) Wound repair and regeneration. Nature 453: 314-321.

Herath TDK, Larbi A, Teoh SH, Kirkpatrick CJ, Goh BT (2018) Neutrophil-mediated enhancement of angiogenesis and osteogenesis in a novel triple cell coculture model with endothelial cells and osteoblasts. J Tissue Eng Regen Med 12: e1221-e1236.

Hicklin DJ, Ellis LM (2005) Role of the vascular endothelial growth factor pathway in tumor growth and angiogenesis. J Clin Oncol 23: 1011-1027.

Jenne CN, Urrutia R, Kubes P (2013) Platelets: bridging hemostasis, inflammation, and immunity. Int J Lab Hematol 35: 254-261.

Liu WC, Chen S, Zheng L, Qin L (2017)Angiogenesis assays for the evaluation of angiogenic properties of orthopaedic biomaterials - a general review. Adv Healthc Mater 6. DOI: 10.1002/adhm.201600434.

Lorenz J, Al-Maawi S, Sader R, Ghanaati S (2018) Individualized titanium mesh combined with platelet-rich fibrin and deproteinized bovine bone: a new approach for challenging augmentation. J Oral Implantol 44: 345-351.

Mandriota SJ, Menoud PA, Pepper MS (1996) Transforming growth factor beta 1 down-regulates vascular endothelial growth factor receptor $2 / \mathrm{flk}-1$ expression in vascular endothelial cells. J Biol Chem 271: 11500-11505.

Martin P (1997) Wound healing - aiming for perfect skin regeneration. Science 276: 75-81.

Miron RJ, Zucchelli G, Pikos MA, Salama M, Lee S, Guillemette V, Fujioka-Kobayashi M, Bishara M, Zhang Y, Wang HL, Chandad F, Nacopoulos C, Simonpieri A, Aalam AA, Felice P, Sammartino G, 
Ghanaati S, Hernandez MA, Choukroun J (2017) Use of platelet-rich fibrin in regenerative dentistry: a systematic review. Clin Oral Investig 21: 1913-1927.

Mukherjee P, Mani S (2013) Methodologies to decipher the cell secretome. Biochim Biophys Acta 1834: 2226-2232.

Nebuloni M, Albarello L, Andolfo A, Magagnotti C, Genovese L, Locatelli I, Tonon G, Longhi E, Zerbi P, Allevi R, Podesta A, Puricelli L, Milani P, Soldarini A, Salonia A, Alfano M (2016) insight on colorectal carcinoma infiltration by studying perilesional extracellular matrix. Sci Rep 6: 22522. DOI: 10.1038/ srep22522.

Nurden AT (2011) Platelets, inflammation and tissue regeneration. Thromb Haemost 105 Suppl 1: S13-33.

Price PJ (2017) Best practices for media selection for mammalian cells. In Vitro Cell Dev Biol Anim 53: 673-681.

Rueden CT, Schindelin J, Hiner MC, DeZonia BE, Walter AE, Arena ET, Eliceiri KW (2017) ImageJ2: ImageJ for the next generation of scientific image data. BMC Bioinformatics 18: 529. DOI: 10.1186/ s12859-017-1934-z.

Simonpieri A, Choukroun J, Del Corso M, Sammartino G, Dohan Ehrenfest DM (2011) Simultaneous sinus-lift and implantation using microthreaded implants and leukocyte- and plateletrich fibrin as sole grafting material: a six-year experience. Implant Dent 20: 2-12.

Soofi SS, Last JA, Liliensiek SJ, Nealey PF, Murphy CJ (2009) The elastic modulus of Matrigel as determined by atomic force microscopy. J Struct Biol 167: 216-219.

Udeabor SE, Adisa AO, Orlowska A, Sader RA, Ghanaati S (2017) Tumor-associated macrophages, angiogenesis, and tumor cell migration in oral squamous cell carcinoma. Ann Afr Med 16: 181-185.

Unger RE, Ghanaati S, Orth C, Sartoris A, Barbeck M, Halstenberg S, Motta A, Migliaresi C, Kirkpatrick CJ (2010) The rapid anastomosis between prevascularized networks on silk fibroin scaffolds generated in vitro with cocultures of human microvascular endothelial and osteoblast cells and the host vasculature. Biomaterials 31: 6959-6967.

Unger RE, Krump-Konvalinkova V, Peters K, Kirkpatrick CJ (2002) In vitro expression of the endothelial phenotype: comparative study of primary isolated cells and cell lines, including the novel cell line HPMEC-ST1.6R. Microvasc Res 64: 384-397.

Vailhe B, Vittet D, Feige JJ (2001) In vitro models of vasculogenesis and angiogenesis. Lab Invest 81: 439-452.

Veronesi F, Borsari V, Sartori M, Orciani M, Mattioli-Belmonte M, Fini M (2017) The use of cell conditioned medium for musculoskeletal tissue regeneration. J Cell Physiol 233: 4423-4442.

Wend S, Kubesch A, Orlowska A, Al-Maawi S, Zender N, Dias A, Miron RJ, Sader R, Booms P, Kirkpatrick CJ, Choukroun J, Ghanaati S (2017) Reduction of the relative centrifugal force influences cell number and growth factor release within injectable PRF-based matrices. J Mater Sci Mater Med 28: 188. DOI: 10.1007/s10856-017-5992-6.

Werner S, Grose R (2003) Regulation of wound healing by growth factors and cytokines. Physiol Rev 83: $835-870$.

\section{Discussion with Reviewers}

Markus Ioibl: Do the authors think similar proangiogenic effects in the EC mono-culture could be obtained by adding recombinant VEGF in the same concentration as measured in the study?

Authors: The proangiogenic effect was, indeed, partly mediated through an increased VEGF expression in the EC-PRF complexes. This was demonstrated during the study and it might lead to the assumption that simply adding recombinant VEGF instead of using PRF could also induce microvessel-like structure formation in EC mono-culture. Nevertheless, to form angiogenic structures, EC mono-culture essentially need a stabilising matrix: either a matrix that is produced by another cell type, e.g. osteoblasts or fibroblasts (Dohle et al., 2010; Dohle et al., 2011; Fuchs et al., 2009), or matrices such as Matrige ${ }^{\circledR}$ or fibringels that are generally used in different angiogenesis arrays (Fuchs et al.,2009; Stahl et al., 2004).

Markus Ioibl: Do the authors consider the effect of PRF as a collective effect of different bioactive molecules or could they name one key player for the promotion of vascularisation?

Authors: We consider the effect of PRF as a collective effect of different bioactive molecules in combination with the fibrin structure, regarding PRF as a unity to study PRF-mediated effects in its entirety. Further studies are currently ongoing separating PRF into its components and analysing their effects individually.

Elena Della Bella: PRF has a pro-angiogenic effect on EC monocultures, but this is not so evident when ECs are co-cultivated with HFs. How could you explain this difference? This should be investigated more in details in the eventuality of a clinical translation of the results.

Authors: The difference in PRF-mediated proangiogenic effect on EC mono-cultures as compared to co-cultures with regard to angiogenic structure formation might be explained by an excessive supply of growth factors, i.e. VEGF and TGF- $\beta 1$, deriving from PRF itself but also additionally released by the HFs. The highest VEGF and TGF- $\beta 1$ concentrations can be found in HF/PRF-complexes after 4 and $7 \mathrm{~d}$. Growth factor concentrations in supernatants of co-culture/PRF-complexes were comparatively lower, supposedly because the growth factors might be consumed by ECs in these complexes. VEGF and TGF- $\beta 1$ are able to induce angiogenic structure formation but might also have opposing effects on ECs since the crosstalk between 
these growth factors also controls EC apoptosis (Hicklin and Ellis, 2005). In general, a co-culture in combination with another factor, such as PRF, is a highly complex system which is similar to the in vivo situation. Therefore, it is usually very difficult to determine exactly which cell type is doing what and how, because the different cell types act together as a unique system and each cell type is influencing the other through paracrine or direct communication, including complicated feedback mechanisms. Nevertheless, we are planning a further study to explain the differences in the angiogenic activation, analysing cell communication and occurring mechanisms in PRF/cell-complexes.

Editor's note: The Scientific Editor responsible for this paper was Mauro Alini. 